\title{
The Structure and Function of Near-Death Experiences: An Algorithmic Reincarnation Hypothesis
}

\author{
Todd Murphy \\ San Francisco, $C A$
}

\begin{abstract}
Hypothesizes that a near-death experience (NDE) is the subjective experience of having the state of consciousness in which a person experiences the last moment of his or her life being turned, in stages, into the state of consciousness experienced as the "point of no return." The life review this, as is interpreted as a review of the states of consciousness experienced during our lives. Our responses to reviewing our own behaviors while in specific states reinforces and classifies them into those to repeat in future lives and those to avoid. We examine a modification of the traditional doctrine of reincarnation that takes into account biological and cultural evolution. This allows an understanding of how the attributes of NDEs could have undergone selection even though all opportunities for mating have already passed at the time of death.
\end{abstract}

KEY WORDS: near-death experience; reincarnation; Buddhism; rebirth.

Seventy percent of near-death experiencers (NDErs) return from their experiences believing in reincarnation (Wells, 1993). Often, they tell of being counseled about the life they lived, and given help in planning their following lives. Not only NDErs, but also a large group of past life regression hypnotherapists (for example, Whitton, 1986) and several major religious traditions accept the doctrine of reincarnation. Ian Stevenson has also uncovered several types of evidence relating

Todd Murphy is an associate researcher with the Behavioral Neurosciences Program at Laurentian University under the direction of Michael A. Persinger, Ph.D. Buddhist Imprimatur granted for this paper by His Holiness Samdech Preah Mahaghoshananda, The Supreme Patriarch of Cambodia. Reprint requests should be addressed to Mr. Murphy at P.O. Box 170414, San Francisco, CA 94117; e-mail: brainsci@jps.net. 
to reincarnation (Stevenson, 1974, 1997). The pressures favoring the exploration of reincarnation as a postulate in the explanation of NDEs are growing. Although polls have found 25 percent of Americans believe in rebirth, the figure reaches nearly 100 percent in other cultures, most importantly in the Hindu and Buddhist worlds.

It should come as no surprise that traditional paradigms for rebirth do not describe NDEs. Those theories were more likely devised to account for experiences during meditation rather than the experience of death. The database of death-related experiences was almost nonexistent, while the database of mystical and transcendent experiences was quite large. The idea was that those who went deeply into meditation were able to see "beyond death's door," and that spiritual practice was a way to defeat death by breaking the cycle of rebirth. Descriptions of meditation experiences were used, it appears, as templates from which speculations about death were traced. Indeed, there is some overlap between the phenomenologies of NDEs and meditation experiences. The theory of rebirth was never formulated to account for NDEs, but some reasonable parameters can be imposed on it, allowing an exploratory hypothesis to be formulated.

One such parameter is derived from the Darwinian theory of natural selection. According to the theory of natural selection, we must be able to explain rebirth as an adaptation that contributed to our survival at some point in the history of our species. If so, then the specific mechanisms by which it operates must be the same for everyone, because we all share a common evolutionary ancestry. The first principle of a Darwinian rebirth hypothesis can be stated thus: Information that enables individuals to survive remains following death in discreet, coherent packets, and other individuals still undergoing prenatal development elsewhere are sensitively dependent upon information in these packets for their development.

A reasonable postulate is: Each person experiences the same state of consciousness prior to the cessation of subjective experience. This implies that the "point of no return" reported by many NDErs, beyond which they felt resuscitation would have been impossible, is a manifestation of a state of consciousness that will eventually appear in each deathprocess unless it is interrupted, often eliciting an NDE report.

Although there appears to be a universal grammar to NDEs, the specific vocabulary of any given case is determined by a variety of factors, including age, culture, the specific circumstances of the person's death, psychological history, and possibly many other factors 
still undiscovered. The specific phenomenology of any particular instance of a "point of no return"-its vocabulary-will be confabulated individually according to these and other factors; but the underlying state of consciousness will be the same.

If the final state of consciousness in each life is the same for everyone, then the NDE must access that state from whatever state of consciousness a person happens to be in at the onset of death, even if it is accompanied by pain or fear. No one knows how or when he or she will die, or what state of consciousness he or she will be in when it happens. The circumstances of death appear at random, and can vary widely from person to person. I propose that NDEs are the subjective experience of having one's randomly-appearing state of consciousness at the time of death brought, in stages, to the nonrandom life review and "point of no return."

\section{Algorithms and NDEs}

Any process that converts randomness to nonrandomness is called an algorithm. According to Daniel Dennett, "An algorithm is a certain sort of formal process that can be counted on-logically-to yield a certain sort of result whenever it is 'run' or instantiated" $(1995$, p. 50). Dennett wrote that there are three key features that characterize an algorithmic process:

"1. substrate neutrality: The procedure for long division works equally well with pencil or pen, paper or parchment, neon lights or skywriting, using any logical system you like. The power of the procedure is due to its logical structure, not just the causal powers of the materials used in the instantiation, just so long as those causal powers permit the prescribed steps to be followed exactly.

2. underlying mindlessness: Although the overall design of the procedure may be brilliant, or yield brilliant results, each constituent step, as well as the transition between steps, is utterly simple. How simple? Simple enough for a dutiful idiot to perform - or for a straightforward mechanical device to perform. The standard textbook analogy notes that algorithms are recipes of sorts, designed to be followed by novice cooks. A recipe book written for great chefs might include the phrase "Poach the fish 
in a suitable wine until almost done," but the algorithm for the same process might begin, "Choose a white wine that says 'dry' on the label; take a corkscrew and open the bottle; pour an inch of wine in the bottom of a pan; turn the burner under the pan on high;..."-a tedious breakdown of the process into deadsimple steps, requiring no wise decisions or delicate judgments or intuitions on the part of the recipe-reader.

3. guaranteed results: Whatever it is that an algorithm does, it always does it, if it is executed without misstep. An algorithm is a foolproof recipe." (Dennett, 1950, pp. 50-51)

NDEs can be seen as demonstrating these features. Substrate neutrality emerges if we assume that the state-specific character of hallucinations also applies to NDEs. Hallucinations are dependent on states of consciousness (Horowitz and Adams, 1970). The same state of consciousness can produce different phenomena in different individuals. The breathtaking variety of NDE phenomena might be manifestations of just a few states of consciousness.

Mardi Horowitz and John Adams (1970) have theorized that the hallucinatory phenomena associated with complex partial seizures arise as expressions of altered states of consciousness. The similarities between NDE phenomena and temporal lobe epileptic symptoms have been noted by several researchers (Saavedra-Aguilar and Gómez-Jeria, 1989; Persinger, 1994). Thus we can reasonably suppose that similar mechanisms might be operating in NDEs and in temporal lobe epilepsy. If so, then it follows that Horowitz and Adams' conclusion might also apply to NDEs. If we choose to make that assumption, then the series of experiences that constitute NDEs is better understood as a series of states of consciousness. The underlying "mindless" apparatus that brings dying persons to the "point of no return" operates on their states of consciousness, and the succeeding experiences are the phenomenological correlates. The bewildering variety of NDE phenomena, as I shall describe below, can be resolved into a few basic states of consciousness, each of which has a specific function.

Underlying mindlessness can be derived from the observation that NDErs find themselves undergoing succeeding stages of their experience automatically. NDErs do not report that they "willed" themselves into the tunnel, for example. That NDEs are outside the control of their experiencers, together with the observation that there 
are significant similarities in many NDEs, suggests that they follow an automatic sequencing.

I have already made Dennett's third feature of algorithmic processes, guaranteed results, a postulate of this model: The guaranteed result is the "point of no return" and eventual rebirth.

\section{Patterns in NDEs}

There seem to be certain grammatical rules governing NDEs. Although the research elucidating them is far from complete, a pattern of rough "rules of thumb" appears to be emerging. Examples include:

1. In India, the death process often begins not with an autoscopic out-of-body experience (OBE) but rather with "seeing" messengers of death whose summons must be answered (Murphy, 2001; Pasricha and Stevenson, 1986). There are, however, some Indian NDEs that begin with an OBE (Blackmore, 1993). The same rule applies to Thai NDEs (Murphy, 2001).

2. Those younger than seven years old often avoid the life review and instead visit heaven or a fairyland (Serdahely, 1990).

3 . In preliterate cultures, the life review is often replaced by a visit to a spirit world in which significant events of the dying person's life manifest symbolically, as features in the spirit world (Kellehear, 1993).

4. NDErs who have been able to anticipate their death and to reflect extensively on their life often do not experience a life review; whereas those whose death appears unexpectedly usually do review their life (Greyson, 1985).

5. NDErs who believe strongly in a particular religious tradition often experience the being of light as they have been taught it appears (Osis and Haraldsson, 1977); whereas atheists may experience it simply as a "presence."

6. NDErs who believe that "all mysteries will be revealed at death" often have a transcendent experience in which mysteries are revealed to their satisfaction (for example, Brinkley and Perry, 1994; Eadie and Taylor, 1992).

7. NDErs who need help, guidance, or an escort during their experience often encounter angels (Lundahl, 1992) or Yamatoots (Murphy, 2001) who may engage the experiencers in long discussions in which their concerns are dealt with. 
8. NDErs who need reassurance that it is all right to be dead often encounter deceased relatives and beloved friends. Joyful reunions with beloved friends who have passed may facilitate positive affect in postmortem states of consciousness. Those too young to have deceased friends but have lost a pet may see the pet instead (Serdahely, 1989-1990). Those who have not lost a pet might see a comforting object such as a toy (Morse, 1994).

9. An NDEr whose life was marked by destructive behavior patterns may experience a life review affectively widened to include the effects of those behaviors on others (Atwater, 1994; Brinkley and Perry, 1994).

This list of "rules of thumb" is both speculative and incomplete. Each item on the list should be regarded as an approximation of a real "grammatical rule" that influences the algorithmic progess of NDEs. It is not possible at present to list a series of rules that will explain the functional connections between specific NDE features and their predisposing factors. Craig Lundahl (1993) delineated a series of NDE rules that addressed the likelihoods of NDE features, but not their functional roles. In any case, it must be emphasized that the rules or axioms that govern NDEs will be applicable not so much to the experiences themselves as to the states of consciousness that produce them.

The different representations of the different phases of NDEs presumably reflect differences in set, age, culture, health, and so on between individuals experiencing the same state. For example, the life review can occur in various NDEs as a serial re-experiencing of one's life, a viewing of multiple television screens (Atwater, 1994), the experience of "watching one's life pass before one's eyes," or a summary of only one's significant life events. These dramatic differences might be accounted for by theorizing that each represents the most efficient phenomenology for the state that produces it for that individual.

\section{Karma and the Life Review}

A feature in every tradition that preserves the doctrine of rebirth or reincarnation is the idea of karma. In order to adopt karma as a legitimate category in building a theory of NDEs, we need to reduce it to its simplest terms. The traditional teachings on karma are filled with 
unfalsifiable implications. Nevertheless, one meaningful statement may be derived from these traditions: Individual behaviors in one life can have an impact on subsequent lives. The theory of natural selection requires that the postulated death and rebirth process should increase chances for survival in some way. That is, if behaviors in one life can influence those of another, that influence must tend to make behaviors in the later life more adaptive. Natural selection has no foresight; adaptations are permanent traits that preserve past expediencies. If we really do reincarnate, we are being reborn not towards nirvana, but rather away from extinction.

For human behavior to become more adaptive it must do so first with respect to our cultural environments, because the evaluation of behavior is both culture-bound and culture-specific. A second principle for a Darwinian rebirth hypothesis emerges and can be stated as follows: States that facilitate adaptive behavior in a given cultural environment in one life tend be repeated in following lives, and states that facilitate maladaptive behavior tend to be avoided.

For the present work, I shall call this the rule of karma. I will call the records of specific states that facilitate or suppress behavior karma. It follows that the function of rebirth may be to pre-adapt us to our cultural environments. However, the conclusion that karmas are reborn in no way implies that human beings are reincarnated, however comforting the idea might be.

In order for karmas to be transmitted from a dying person to an infant or fetus, they must be broadcast in some way. It seems reasonable to suppose that karma of a living person differs from that of a person who has gone past the "point of no return" in the way it is stored-although not in the information it contains. A piece of computer software contains the same information whether it is stored on a disk or actually being used in the computer's microprocessor. Likewise, the recorded states of consciousness are the same whether the karmic records are in our brains or downloading to a subsequent birth. The "point of no return" might be likened to the point at which a computer program is transferred from its random-access memory (RAM) to a disk. The life review, I suggest, is the phenomenological manifestation of a state of consciousness that creates, from the effects of states of consciousness experienced in one lifetime, suggestions for states that enable adaptive behavior during the next life.

The life review can lead NDErs to re-examine everything they have ever done, not as they remember their experiences, but approximately 
as they actually happened. When they remember having done something adaptive, and that recollection induces positive affect, the correlative state is marked for repetition in their next life. When they remember having done something maladaptive, and that recollection makes them feel bad, the state is marked for suppression in their next life. Because it is one's culture that designates what is good or bad, the possibility arises that the life reviews sorts states according to how likely they are to generate culturally adaptive behavior. The life review must focus on behavior because behaviors are state-specific; states of consciousness cannot be viewed directly, but the behaviors that act them out can. Re-experiencing an event will invoke the state one was in at the time of the event.

\section{Culture and NDEs}

The reason so many NDEs features are culture-bound may be that the death process is an evolutionary adaption favoring those who "take rebirth," specifically by enhancing their ability to gain status in the complex cultures that appeared in our recent evolution. Individuals who cannot follow the rules do not acquire much status. Karma, I suggest, is a set of positively and negatively reinforced states of consciousness that enable adaptive behaviors. Having karma might

give individuals an advantage not only in surviving but also in following cultural rules, eliminating an important obstacle to achieving rank. Individuals with high status have better chances of producing offspring than those with low status. The effects of rebirth would be amplified over time if the first individuals 'taking' rebirth consistently rose to the top of the social ladder. I suggest that those who had life reviews at the end of one life were more likely to be reborn to become dominant alpha individuals, with better mating opportunities than betas and deltas. The culture-bound character of NDEs could be a case of form following function. Karma can change as culture changes. A state approved in one cultural context might be avoided following cultural change.

\section{"Hellish" NDEs}

One important pattern emerging is that some NDEs involve profound negative affect. These "hellish" experiences bear striking structural similarities to positive ones. P. M. H. Atwater commented: "During my 
own interviews of experiencers... I discovered little difference between heavenly and hellish near-death episodes in consideration of how elements unfolded in sequence" (1994, p. 40).

Lysergic acid diethylamide (LSD), which usually induces a pleasant experience, can also produce a negative or hellish ordeal. Because the same chemical can lead to both types of experience, it seems unlikely that any specific affect is integral to the LSD experience. The same is true for seizures in temporal lobe epilepsy (LaPlante, 1993). If Horowitz and Adams' (1970) hypothesis applies to NDEs as it does to LSD, then the possibility that negative affect is integral to unpleasant NDEs can be reasonably ruled out. The best candidate for the cause of hellish NDEs in our model is resistance, as I shall describe below.

\section{Neurology}

The source of the hellish affect is another question. Temporal lobe research by Michael Persinger (1994) suggests that single states of consciousness can evoke very different affects and implies that temporal lobe affects may be implicated in both hellish and blissful NDEs.

The involvement of the brain's temporal lobes in NDEs appears to be well established. Persinger (1987) has theorized that when our species first evolved its unique cognitive abilities, two parts of our brains enlarged disproportionately: the frontal lobes, generally specialized for extrapolating into the future, and the temporal lobes, generally specialized for remembering the past. Those developments allowed people to remember death and to realize that the same thing would happen to them in the future. That upgrade included a software for death anxiety. However, the adaptive value of being able to project into the future, to imagine ways of dying and so avoid them, would have been canceled out by the dysphoria it would also have produced. A compensatory mechanism seems to have appeared at the same time. Arnold Mandell speculated on "affective specialization in the lateralization of the brain, with 'negative' emotions like fear and paranoia and dysphoric feelings like sorrow and depression lying ... [in] ... the left temporal lobe, and with the mute, geometrically cognitive, musical right temporal lobe specialized for joy" (1980, p. 411). Mandell's argument refers to the temporal cortex. Melvin Morse contends that portions of the right temporal lobe mediate the entire NDE experience, calling it the "circuit boards of mysticism" (Morse and Perry, 1992). The left temporal cortex's functions, on the other hand, 
include profound negative affect. Wilder Penfield and Herbert Jasper, discussing the results of electrical stimulation of the temporal lobe surface, described unpleasant emotions:

These ictal emotions which patients described as fear, fright, scared feeling, terror, sadness, loneliness ... [which]... may be said to have ganglionic representation within the fissure of Sylvius and their underlying circular sulcus... The ictal emotion is produced as a distinct experience and is the result of localized cortical discharge. (Penfield and Jasper, 1954, p. 451).

Illustrations accompanying this text showed that it referred to the left temporal cortex.

These findings suggest that the reason some NDEs are hellish is that the positive affect of most NDEs, originating in the right temporal cortex, is replaced by negative affect from the left temporal cortex. This hypothesis, if true, it might explain how an NDE can be unpleasant, but not why it is so.

\section{Psychology}

I propose that one reason some NDEs are unpleasant is that the person is resisting the death process. Atwater (1994) suggested that unpleasant NDEs are related to an individual history of repressed guilt, while Bruce Greyson and Nancy Bush's (1992) accounts of distressing NDEs all contain comments to the effect that the person did not want to die. The most commonly reported emotion in unpleasant NDEs is fear. Whether the crucial factor is guilt or fear, both feelings inspire resistance. This idea, together with the algorithmic interpretation of NDEs I am proposing here, implies that a specific affective state must be achieved. That affective state, I suggest, is surrender, the opposite of resistance. I do not propose that surrender must be achieved only to experience the "point of no return"; rather, I suggest that it also might enable the life review. In this interpretation of NDEs, each phenomenon is an expression of a state of consciousness, and each state of consciousness has the specific function of increasing the likelihood of reaching first the life review, and then the "point of no return." The life review is less anomalous as a result of natural selection, if it is seen as contributing to the success of those who undergo rebirth.

If all NDEs not interrupted by resuscitation go through some form of the life review, then I suggest that any negative affect must be changed to a positive one before life review is achieved. The life review can require that a person examine, re-experience, or witness his or 
her own maladaptive behavior. When this same thing happens in psychotherapy, it often evokes resistance. Resistance in psychotherapy often occurs in conjunction with negative affect; if it were to occur during the death process, the experience might also be unpleasant. As noted above, Atwater (1994) suggested that hellish NDEs are a result of repressed guilt, while Greyson and Bush's (1992) cases of distressing NDEs all contained allusions to not wanting to die. Both of these mechanisms implicated in hellish NDEs may be grouped together as resistance. Resistance hinders meaningful self-examination in psychotherapy; perhaps it does the same during life reviews.

Hell: Special case \#1. Not all negative NDEs are necessarily the product of resistance. A different type of negative affect might arise in conjunction with the life review, as in the case recounted by a former Vietnam War assassin (Brinkley and Perry, 1994). The following narrative suggests a symbolic, aversive life review:

I was unconscious to all onlookers, yet something weird was happening to me.... I was in a circle of light. I looked down upon the accident scene.... I looked into my car and saw myself trapped and unconscious. I saw several cars stop and a lady taking my children to her car to sit and rest until the ambulance would arrive.... A hand touched mine, and I turned to see where this peace and serenity and blissful feeling was coming from ... and there was Jesus Christ-I mean the way he is made out to be in all the paintings-and I never wanted to leave this man and this place.

I was led around to a well, because I wanted to stay with him and hold his hand. He led me from a side of bliss to a side of misery. I did not want to look, but he made me look-and I was disgusted and horrified and scared ... it was so ugly. The people were blackened and sweaty and moaning in pain and chained to their spots. And I had to walk through the area back to the well. One was even chained to the evil side of the well... I wanted them to help him, but no one would-and I knew that I would be one of these creatures if I stayed. I hated it there. I couldn't wait to get to the well and go around it. He led me to it, but he made me go through it alone as he watched....

I leaned over the well... There were three children calling "Mommie, Mommie, Mommie, we need you. Please come back to us." .. The little girl looked up at me and begged me to go back to life-and then all at once... I saw the accident scene again.... (Greyson and Bush, 1992, pp. 105-106)

The aversive episode in this NDE centers around images of children. It is possible that the woman in this case wanted to have a child, or more children, and saw her life in terms of a hell in which she 
was to rescue the souls of unborn children by bringing them into the world. This unusual life review makes sense if we assume that karma functions to increase adaptive behavior from one life to the next, and that during most of our evolutionary history raising a child is one of the most adaptive things a woman could do. Even though her review was confined to only one assumed behavior-being childless-resolving only that one karma in her next life by having a child, and thus passing on her genetic material, would have increased dramatically the adaptiveness of her behavior as a result of this review.

Hell: Special case \#2. One phenomenon that does not appear in typical, pleasant NDEs is the experience of a vacuum. Although the word void has been used to describe it, it does not seem to be the same as the void that occurs in the stage along with the tunnel. That void associated with the tunnel often induces highly positive affect, while the vacuum is, as far as I am aware, always hellish. One experiencer described the vacuum as follows:

It was empty... and dark. Not like night dark, somehow, it was thinner-whatever that means. It was very dark and immense all around, but somehow I could see them [tormenting beings]; the voidness seemed to thin out somewhere off by the horizon,... but it wasn't lighter, just thinner. It seemed to go on forever. ... That utter emptiness just went on and on.... there didn't seem to be any end of it, and no way out. (Greyson and Bush, 1992, p. 102)

Other descriptions include: "suspended in a total vacuum with nothing to see or do for eternity" (Greyson and Bush, 1992, p. 103) and "hours went on with absolutely no sensation...there was no hot, no cold, no light, no taste, no smell, no sensation whatsoever, none, other than ... a slight sensation of travelling.... it became unbearable, it became horrific" (Greyson and Bush, 1992, p. 104).

The majority of cases of the vacuum in Greyson and Bush's study occurred during childbirth under anesthesia. Perhaps pregnancy provides a "fail-safe" mechanism that delays the onset of the "point of no return" until the last possible moment. Such an adaptation would greatly enhance the chances for survival of infants born to mothers who hemorrhaged during childbirth. Deaths due to violent trauma or old age were probably less likely to end in resuscitation than those in childbirth. Historically, a common cause of death in childbirth has been loss of blood; but blood can be replenished easily, and death thereby easily reversed. An NDE "fail-safe" mechanism for childbirth would increase the chances for survival not only for the NDEr but for her child as well. 
Like most NDE phenomena, the vacuum does not occur only at death. It has also been found in sleep paralysis, a neurological disorder that affects the ability to wake up (LaPlante, 1993). In these cases, the victims find themselves suspended in a vacuum, trying to wake up but unable to. Because both sleep and death involve moving through different states of consciousness, symptoms occurring in sleep disorders might also appear in NDEs. If so, the vacuum, occurring in both, could be the phenomenon confabulated out of the inability or unwillingness to move from one state to another. That hypothesis suggests an interesting possibility: that the adaptation that created the death process was a new application of the neurological mechanisms that previously had been responsible only for sleep. Both processes involve multiple, sequenced states of consciousness. This speculation is lent credence by reports of NDEs induced by lucid dreaming (Green, 1995; Rogo, 1990).

\section{Speculations on the Functions of Typical Phase of NDEs}

Death can often be anticipated in protracted terminal illness, and that anticipation can have an impact on the death process. Several researchers have demonstrated increased incidence of spontaneous altered states in the period shortly before a person approaches death (Greyson, 1985; Morse and Perry, 1994; Osis and Haraldsson, 1977). Dying patients not yet at the brink of death frequently report seeing "the light" in their rooms, and visitations by angels and beloved dead friends and relatives. The appearance of NDE phenomenology in these premortem periods implies that the same states of consciousness that appear during the death process can begin to operate whenever a person becomes cognizant that his or her life will end. When people who have already experienced these states of consciousness begin their death process, they will be familiar with and less likely to resist many of the percepts they encounter.

The OBE at the beginning of many NDEs might function to convince the dying person that he or she is dead. Through much of our evolutionary history, deaths were often traumatic. Males often died violently, during war or on hunts. Women, as we tend to forget in our safer, modern times, often died in childbirth. Autoscopic OBEs would permit the experiencers to look back on themselves and see a very distressed corpse.

In Thailand and India, on the other hand, NDEs are more likely to commence with a visitation by a Yamatoot, a messenger of Yama, the 
lord of the dead (Murphy, 2001; Pasricha and Stevenson, 1986), than with an OBE. Resistance to the summons of a Yamatoot is futile. Fear of Yamatoots occurred frequently in Karlis Osis and Erlendur Haraldsson's (1977) study of premortem states in India. It is possible that the incidence of distressing NDEs varies from one culture to the next. Within Western culture, it is also possible that in past centuries, when many more death processes were hellish than at present (Greyson and Bush, 1992), the Grim Reaper served as a Western analog of a Yamatoot, and that Europeans once ran from the Grim Reaper as Hindus sometimes run from the Yamatoots sent to take them.

The typical "core" NDE progresses to the tunnel or void after an autoscopic OBE, in which one continues to sense an environment. These experiences might indicate that sensory perception has ended, and that the defense mechanisms valid in states that accompany sensory perception are now obsolete. The symbolism of the tunnel as a transition from one "world" to another in NDEs has already been explored (Chari, 1982). Perhaps the tunnel or void reflects a state of consciousness that functions to take one from sensory perception to wholly endogenous percepts.

The appearance of dead beloved friends and relatives might evoke the feeling that it is all right to be dead. Feelings of loneliness, separation anxiety, feelings of being abandoned, guilt at leaving those who are dependent on us, or grief at the loss of beloved living people might be mollified by the creation of an inner, death-contextualized, social environment. One could feel safe there and thus be less likely to resist out of fear.

The being of light, which typically precedes or appears at the same time as the life review, might function to prevent resistance. The allpervading love and feelings of acceptance the being of light evokes is incompatible with the negative affect created by resistance.

\section{Conclusion}

NDEs can be viewed as an algorithmic process that alters the many states of consciousness possible at the time of death so as to produce first, the life review, and then the "point of no return." There can be positive or negative affect in any near-death state of consciousness, although the states will tend toward the positive affect that decreases resistance to the experience. I propose that the life review has a special function: to sort out behaviors to repeat in future lives from those to avoid. 
An enormous amount of work needs to be done for the present hypothesis to be developed into a theory. The list of grammatical "rules of thumb" needs to find a more rigorous expression, and it needs to be expanded to include other rules, most obviously those culturebound effects specific to major cultural groups. Some age-specific NDE features have been noted, but there may be features characteristic of specific phases of life: Are there common elements in the NDEs of newlyweds, of adolescents, of pregnant women? A classification system for the factors that influence the sucession of states of consciousness needs to be devised and tested, beyond the obvious factors of age, culture, psychological set at time of death, expectations regarding what death will feel like, and the length of time one has to anticipate one's death. When we consider how young and how poorly funded NDE research is, it seems reasonable to assume that there are determinative influences still waiting to be discovered.

\section{Predictions and Applications}

Before considering the potential applications of my hypothesis, we should note that the validity of an hypothesis is determined not by its initial applicability but rather by its falsifiability. One prediction of the present hypothesis is that NDEs of all cultures will exhibit a typical sequence. The notion that karma is a set of states of consciousness, coupled with observations that there are magnetic components and/or bases for states of consciousness (Persinger, 1994), predicts that the brain might emit magnetic signals at some point in the death process. If these signals are propagated within the earth's magnetic field, then we are left with the prediction that the intensity of these signals should be within the range of the constant (steady-state) values for the geomagnetic field (Persinger, 1995). If so, then the geomagnetic field might serve as the medium for karmic signals. Interactions between geomagnetic phenomena and NDE experiences have been noted in the literature (Persinger, 1995). Failure to find such signals emitted from a dying brain (once we have developed the technology to read them) would cast doubt on my hypothesis.

A research implication of the algorithmic NDE hypothesis is that NDE phenomena need to be catalogued in order to provide a database that can allow researchers to analyze and classify the states of consciousness involved in dying. Eventually, perhaps, therapists might devise treatments for the post-NDE personality syndrome (Atwater, 1988) directed not at the aftereffects of NDEs but rather at the 
aftereffects of the specific states of consciousness experienced during specific events. The understanding that death will involve multiple states of consciousness, each one played out as a distinct experience with deeply meaningful content, may reduce death anxiety in those terminally ill people who experience premortem altered states of consciousness. For example, understanding the functions of the various kinds of hellish NDEs might lead to techniques in premortem psychology that would allow the identification of those at risk and counseling to reduce their risk. If premortem and postmortem altered states are as similar as their similar phenomenologies imply, then the affects associated with those states before death will be the ones most likely to occur after death.

One final distant possibility, so improbable as to invite dismissal by many scientists, could nevertheless produce vast benefits for humanity. Purportedly miraculous cures are sometimes reported following NDEs. Terminally ill patients sometimes report that they were visited by an angel who told them they were healed, and thereafter there was no sign of a tumor or their T-cell counts rose dramatically. Isolation of the state of consciousness associated with this phenomenon and identification of its manifestations and predisposing factors might open up avenues of research into "miraculous" cures. Recognition that such miracles are correlates of specific states of consciousness might allow common factors associated with such cures to be discerned, which might in turn suggest ways to induce those states in a clinical setting. Chinese medical tradition has recorded many such cures associated with poisonous mushrooms, some of which, in small enough doses, act as hallucinogens (Bernard Yeh, personal communication, 1988). Many NDE phenomena have been induced in a laboratory setting by the application of low-intensity complex magnetic signals to the temporal lobes (Ruttan, Persinger, and Koren, 1990). If the state of consciousness in which miraculous cures occur can be induced by stimulation of the temporal lobes with magnetic signals, the induction of such states could be added to the tools of modern medicine.

\section{References}

Atwater, P. M. H. (1988). Coming back to life: The after-effects of the near-death experience. New York, NY: Dodd, Mead.

Atwater, P. M. H. (1994). Beyond the light: What isn't being said about the near-death experience. New York, NY: Birch Lane Press. 
Blackmore, S. J. (1993). Near-death experiences in India: They have tunnels too. Journal of Near-Death Studies, 11, 205-217.

Brinkley, D., and Perry, P. (1994). Saved by the light: The true story of a man who died twice and the profound revelations he received. New York, NY: Villard.

Chari, C. T. K. (1982). Parapsychological reflections on some tunnel experiences. Anabiosis: The Journal of Near-Death Studies, 2, 110-131.

Dennett, D. C. (1995). Darwin's dangerous idea. New York, NY: Simon and Schuster.

Eadie, B. J., and Taylor, C. (1992). Embraced by the light. Placerville, CA: Gold Leaf Press.

Green, J. T. (1995). Lucid dreams as one method of replicating components of the neardeath experience in a laboratory setting. Journal of Near-Death Studies, 14, 49-59.

Greyson, B. (1985). A typology of near-death experiences. American Journal of Psychiatry, $142,967-969$.

Greyson, B., and Bush, N. E. (1992). Distressing near-death experiences. Psychiatry, 55, 95-110.

Horowitz, M. J., and Adams, J. E. (1970). Hallucinations on brain stimulation: Evidence for revision of the Penfield hypothesis. In W. Keup (Ed.), Origin and mechanisms of hallucinations (pp. 13-22). New York, NY: Plenum Press.

Kellehear, A. (1993). Culture, biology, and the near-death experience: A reappraisal. Journal of Nervous and Mental Disease, 181, 148-156.

LaPlante, E. (1993). Seized. New York, NY: HarperCollins.

Lundahl, C. R. (1992). Angels in near-death experiences. Journal of Near-Death Studies, $11,49-56$.

Lundahl, C. R. (1993). The near-death experience: A theoretical summarization. Journal of Near-Death Studies, 12, 105-118.

Mandell, A. J. (1980). Toward a psychobiology of transcendence: God in the brain. In J. M. Davidson and R. J. Davidson (Eds.), The psychobiology of consciousness (pp. 379-464). New York, NY: Plenum.

Morse, M. L. (1994). Near-death experiences and death-related visions in children: Implications for the clinician. Current Problems in Pediatrics, 24, 55-83.

Morse, M. L., and Perry, P. (1992). Transformed by the light: The powerful effects of near-death experiences on people's lives. New York, NY: Villard.

Morse, M. L., and Perry, P. (1994). Parting visions: Uses and meanings of pre-death, psychic, and spiritual experiences. New York, NY: Villard.

Murphy, T. (2001). Near-death experiences in Thailand. Journal of Near-Death Studies, $19,161-178$.

Osis, K., and Haraldsson, E. (1977). At the hour of death. New York, NY: Avon.

Pasricha, S., and Stevenson, I. (1986). Near-death experiences in India: A preliminary report. Journal of Nervous and Mental Disease, 174, 165-170.

Penfield, W., and Jasper, H. (1954). Epilepsy and the functional anatomy of the human brain. Boston, MA: Little, Brown.

Persinger, M. A. (1987). Neuropsychological bases of God beliefs. New York, NY: Praeger.

Persinger, M. A. (1994). Near-death experiences: Determining the neuroanatomical pathways by experiential patterns and simulation in experimental settings. In L. Bessette (Ed.), Le processus de guérison: Par-delà la souffrance ou la mort [Healing: Beyond suffering and death] (pp. 277-286). Beauport, Canada: MNH Publications.

Persinger, M.A. (1995). Out-of-body experiences are more probable in people with elevated complex partial epileptic-like signs during periods of enhanced geomagnetic activity. Perceptual and Motor Skills, 80, 563-569.

Rogo, D. S. (1990). An experimentally-induced NDE [Letter]. Journal of Near-Death Studies, 8, 257-260.

Ruttan, L. A., Persinger, M. A., and Koren, S. (1990). Enhancement of temporal loberelated experiences during brief exposures to milligause intensity extremely low frequency magnetic fields. Journal of Bioelectricity, 9, 33-54. 
Saavedra-Aguilar, J. C., and Gómez-Jeria, J. S. (1989). A neurobiological model for neardeath experiences. Journal of Near-Death Studies, 7, 205-222.

Serdahely, W. J. (1989-90). A pediatric near-death experience: Tunnel variants. Omega, $20,55-62$.

Serdahely, W. J. (1990). Pediatric near-death experiences. Journal of Near-Death Studies, 9, 33-39.

Stevenson, I. (1974). Twenty cases suggestive of reincarnation. Charlottesville, VA: University of Virginia Press.

Stevenson, I. (1997). Reincarnation and biology: A contribution to the etiology of birthmarks and birth defects. Westport, CT: Praeger.

Wells, A. D. (1993). Reincarnation beliefs among near-death experiencers. Journal of Near-Death Studies, 12, 17-34.

Whitton, J. L. (1986). Life between life. New York, NY: Warner. 